\title{
Nambu-Goldstone dynamics and generalized coherent-state functional integrals
}

\author{
Massimo Blasone ${ }^{1}+\frac{1}{\dagger}$ and Petr Jizba' $\mathbb{S}$ \\ ${ }^{1}$ INFN, Gruppo Collegato di Salerno and Universita' di Salerno, Via Ponte don \\ Melillo, 84084 Fisciano (SA), Italy \\ ${ }^{2}$ FNSPE, Czech Technical University in Prague, Břehová 7, 11519 Praha 1, Czech \\ Republic
}

\begin{abstract}
The present paper gives a new method of attack on the Nambu-Goldstone dynamics in spontaneously broken theories. Since the target space of the NambuGoldstone fields is a group coset space, their effective quantum dynamics can be naturally phrased in terms of generalized coherent-state functional integrals. As an explicit example of this line of reasoning we construct a low-energy effective Lagrangian for the Heisenberg ferromagnet in broken phase. The leading field configuration in the WKB approximation leads to the Landau-Lifshitz equation for quantum ferromagnet. The corresponding linearized equations allow to identify the Nambu-Goldstone boson with ferromagnetic magnon.
\end{abstract}

Keywords: Nambu-Goldstone bosons, Coherent-state functional integrals, Non-linear $\sigma$ models, Landau-Lifshitz magnons

$\ddagger$ Corresponding author: blasone@sa.infn.it

$\mathbb{S}$ Corresponding author: p.jizba@fjfi.cvut.cz 


\section{Introduction}

Functional integrals provide indisputably a powerful tool in diverse areas of physics, both computationally and conceptually. They often offer the easiest route to derivation of perturbation expansions, accommodate naturally gauge symmetry and serve as an excellent framework for non-perturbative analysis [1, 2]. Growing popularity among practitioners in both high-energy and solid-state physics enjoy functional integrals which are based on the occupation number representation or on the Fock space. In contrast, the functional integrals that are rooted in the over-complete set of coherent states (CS) are used comparatively less. Despite their cleaner mathematical structure are the CS-based functional integrals still rather interesting curiosity than full-fledged tools of particle or solid-states physics.

It is purpose of this paper to call attention to the fact that CS-based functional integrals constructed from the so-called group-related or generalized CS [3, 4, 5, 6, 7] offer a very natural tool in theory of critical phenomena with genuine phenomenological implications. In particular, they have a built-in quality to describe an effective low-energy behavior of systems with spontaneous breakdown of a global continuous symmetry provided the interest lies in the low-energy gapless excitations known as Nambu-Goldstone (NG) bosons. We will illustrate our point by employing the generalized CS functional integrals to investigate the low-energy behavior of ferromagnets in the broken phase, i.e., below the Curie temperature.

The structure of the paper is as follows. To set the stage we recall in the next section some fundamentals of the group-related CS with a special emphasis on the $S U(2)$ CS. Section 3 is devoted to formulation of functional integrals by means of generalized CS. A natural appearance of the geometric Berry-Anandan phase in the action of the CS functional integrals and the way how it may affect the dynamics is also discussed. As an explicit example we derive the $S U(2)$ CS functional integral. The rôle of the group quotient space as an arena for the dynamics of NG fields is discussed in Section 4. There we also prove the NG theorem with the help of the cosetspace construction of spontaneous symmetry breakdown (SSB). Distinction between relativistic and non-relativistic versions of the NG theorem is stressed. In Section 5 we observe that transition amplitudes as well as partition function for NG modes can be formulated via the generalized CS functional integrals. To put more flesh on the bare bones we investigate the low-temperature properties of the quantum Heisenberg model of ferromagnet in a broken phase. The corresponding CS functional integral can be identified with the $S U(2) / U(1)-\sigma$ model. The WKB approximation yields in the limit of continuous spin lattice (i.e., large wavelength limit) Landau-Lifshitz equations for quantum forromagnet. Linearized version of the latter equations allows to identify the NG field with the massless spin wave. The NG boson then corresponds to ferromagnetic magnon. Various remarks and generalizations are postponed to the concluding section. 


\section{Group-related coherent states}

To construct the CS related to a Lie group $G$ we follow here Ref. [3]. Let $\hat{D}(g), g \in G$ be an irreducible unitary representation of $G$ acting in some Hilbert space $\mathcal{H}$. We choose a normalized fiducial state vector in $\mathcal{H}$ and denote it as $|0\rangle$. The generalized CS's corresponding to $G$ are then defined as

$$
|0(g)\rangle=\hat{D}(g)|0\rangle \quad \text { for } \forall g \in G .
$$

With foresight of applications in the SSB theory, we have denoted the group-related CS as $|0(g)\rangle$. Two CS $\left|0\left(g_{1}\right)\right\rangle$ and $\left|0\left(g_{2}\right)\right\rangle$ represent the same physical state in $\mathcal{H}$ if

$$
\hat{D}\left(g_{1}\right)|0\rangle=e^{i \alpha\left(g_{1}, g_{2}\right)} \hat{D}\left(g_{2}\right)|0\rangle \Leftrightarrow \hat{D}\left(g_{2}^{-1} g_{1}\right)|0\rangle=e^{i \alpha\left(g_{1}, g_{2}\right)}|0\rangle .
$$

Defining the stability group $H_{|0\rangle}$ as a group of transformations leaving $|0\rangle$ invariant (up to a phase), i.e.,

$$
H_{|0\rangle}=\left\{h \in G: \hat{D}(h)|0\rangle=e^{i \beta(h)}|0\rangle, \beta(h) \in \mathbb{R}\right\},
$$

the distinct $G$-related CS can be parameterized by elements of the coset $G / H_{|0\rangle}$. Since $H_{|0\rangle}$ 's for different fiducial states are mutually isomorphic subgroups of $G$ we will simply use $H$ instead of $H_{|0\rangle}$.

Let $d \mu(g)$ be the left-invariant group measure, i.e., for any fixed $g_{0} \in G, d \mu\left(g_{0} \cdot g\right)=$ $d \mu(g)$. Having $d \mu(g)$, the measure on the coset space $G / H$ is naturally induced. We denote it as $d \boldsymbol{\zeta}$. The resolution of the unity can be then written as

$$
\hat{\mathbf{1}}=c \int_{G} d \mu(g)|0(g)\rangle\left\langle 0(g)\left|=c \int_{G / H} d \boldsymbol{\zeta}\right| 0(\boldsymbol{\zeta})\right\rangle\langle 0(\boldsymbol{\zeta})|
$$

Here $c$ is determined so as to fulfill the consistency condition

$$
1=\left\langle 0\left(\boldsymbol{\zeta}^{\prime}\right) \mid 0\left(\boldsymbol{\zeta}^{\prime}\right)\right\rangle=c \int_{G / H} d \boldsymbol{\zeta}\left|\left\langle 0\left(\boldsymbol{\zeta}^{\prime}\right) \mid 0(\boldsymbol{\zeta})\right\rangle\right|^{2}, \quad \boldsymbol{\zeta}^{\prime} \in G / H
$$

It is thus meaningful to restrict oneself to representations $\hat{D}(g)$ that are square integrable over the quotient $G / H$. More up-to-date view on the group-related CS together with much of the background material can be found, for instance, in Refs. [8, 9].

\subsection{SU(2) coherent states}

For our purpose we will specifically consider the $S U(2)$ CS. The $S U(2)$ group has three generators $\hat{J}_{1}, \hat{J}_{2}, \hat{J}_{3}$ which close the $s u(2)$ algebra

$$
\left[\hat{J}_{+}, \hat{J}_{-}\right]=2 \hat{J}_{3} \quad\left[\hat{J}_{3}, \hat{J}_{ \pm}\right]= \pm \hat{J}_{ \pm} .
$$

Here $\hat{J}_{ \pm}=\hat{J}_{1} \pm i \hat{J}_{2}$. The unitary irreducible representations of the $s u(2)$ algebra are finite-dimensional and are spanned by states $|j, m\rangle$ fulfilling

$$
\begin{aligned}
& \hat{J}_{3}|j, m\rangle=m|j, m\rangle, \\
& \hat{J}_{ \pm}|j, m\rangle=\sqrt{(j \mp m)(j \pm m+1)}|j, m \pm 1\rangle, \quad(|m| \leq j) .
\end{aligned}
$$


The representations of $S U(2)$ are labeled by the eigenvalues of the $s u(2)$ Casimir operator:

$$
\hat{\mathcal{C}}=\hat{\mathbf{J}}^{2}=\frac{1}{2}\left(\hat{J}_{+} \hat{J}_{-}+\hat{J}_{-} \hat{J}_{+}\right)+\hat{J}_{3}^{2}=j(j+1) \hat{\mathbf{1}}
$$

i.e.,

$$
\hat{\mathbf{J}}^{2}|j, m\rangle=j(j+1)|j, m\rangle \quad \text { with } \quad j=0, \frac{1}{2}, 1, \frac{3}{2}, \ldots
$$

As the fiducial vector we might choose the state $|j,-j\rangle$. In this way each representation has its unique fiducial state — "vacuum state" $|0\rangle \equiv|j,-j\rangle$. The stability group is the subgroup of rotations around the $z$-axis, thus $H=U(1)$. According to Eq. (44) the distinct CS are labeled by $\zeta \in G / H$. By noting that $S U(2) / U(1) \cong \mathcal{S}^{2}$ we can identify $\zeta$ with the spherical angles $\theta$ and $\varphi$. The associated CS can then be written as $|0(\theta, \varphi)\rangle$ :

$$
|0(\theta, \varphi)\rangle=\hat{D}(\theta, \varphi)|0\rangle=\exp [i \theta(\hat{\mathbf{J}} \cdot \mathbf{n})]|0\rangle,
$$

with the unit vector $\mathbf{n}=(\sin \varphi, \cos \varphi, 0)$. Using the Gauss decomposition formula

$$
\hat{D}(\theta, \varphi)=e^{\xi \hat{J}_{+}} e^{\log \left(1+|\xi|^{2}\right) \hat{J}_{3}} e^{-\xi^{*} \hat{J}_{-}}, \quad \xi=\tan \frac{\theta}{2} e^{i \varphi},
$$

one can alternatively use the more economical form

$$
|0(\theta, \varphi)\rangle=\left(1+|\xi|^{2}\right)^{-j} e^{\xi \hat{J}_{+}}|0\rangle \equiv|0(\xi)\rangle \text {. }
$$

The scalar product of two CS $|0(\xi)\rangle$ can be written in the form

$$
\left\langle 0\left(\xi^{*}\right) \mid 0(\xi)\right\rangle=\frac{\left(1+\xi^{*} \xi\right)^{2 j}}{\left(1+\left|\xi^{\prime}\right|^{2}\right)^{j}\left(1+|\xi|^{2}\right)^{j}} .
$$

An important implication of Eq. (13), that will be relevant later, is that

$$
\left|\left\langle 0\left(\xi^{\prime *}\right) \mid 0(\xi)\right\rangle\right|^{2}=\left(\frac{1+\mathbf{m}^{\prime} \cdot \mathbf{m}}{2}\right)^{2 j}
$$

Here $\mathbf{m}=(\sin \theta \cos \varphi, \sin \theta \sin \varphi, \cos \theta)$ is the unit vector parameterizing $\mathcal{S}^{2}$. Analogous arguments hold also for $\mathbf{m}^{\prime}$. Since the $S U(2)$ CS can be equally well parametrized by $\mathbf{m}$ we will use the notation $|0(\mathbf{m})\rangle \equiv|0(\xi)\rangle=|0(\theta, \varphi)\rangle$. According to Eq. (4) the resolution of the unity reads

$$
\hat{\mathbf{1}}=\int_{S U(2)} d \mu(g)|0(g)\rangle\left\langle 0(g)\left|=c \int_{\mathcal{S}^{2}} d \mathbf{m}\right| 0(\mathbf{m})\right\rangle\langle 0(\mathbf{m})| .
$$

The constant $c$ is determined from the normalization condition

$$
1=c \int_{\mathcal{S}^{2}} d \mathbf{m}\left|\left\langle 0\left(\mathbf{m}^{\prime}\right) \mid 0(\mathbf{m})\right\rangle\right|^{2}=c \frac{4 \pi}{2 j+1} .
$$

So finally the resolution of the unity may be written in one of the following equivalent forms:

$$
\hat{\mathbf{1}}=\frac{2 j+1}{4 \pi} \int_{\mathcal{S}^{2}} d \mathbf{m}|0(\mathbf{m})\rangle\left\langle 0(\mathbf{m})\left|=\frac{2 j+1}{\pi} \int_{\mathcal{S}^{2}} \frac{d \xi d \xi^{*}}{\left(1+|\xi|^{2}\right)^{2}}\right| 0\left(\xi^{*}\right)\right\rangle\langle 0(\xi)|,
$$

where in the last line we have used

$$
d \xi d \xi^{*} \equiv d \Re \xi d \Im \xi
$$

with $\Re$ and $\Im$ denoting the real and imaginary parts, respectively. 


\section{3. $S U(2)$ CS functional integral}

\subsection{Generalized coherent states and functional integrals}

We are now in position to construct the corresponding functional-integral representation of a transition amplitude $\left\langle 0\left(\boldsymbol{\zeta}_{f}\right), t_{f} \mid 0\left(\boldsymbol{\zeta}_{i}\right), t_{i}\right\rangle$. Similarly as in the usual functional-integral constructions [1] the key is the Heisenberg-picture resolution of unity that in the present case reads (cf Eq. (4))

$$
\hat{\mathbf{1}}=c \int_{G / H} d \boldsymbol{\zeta}|0(\boldsymbol{\zeta}), t\rangle\langle 0(\boldsymbol{\zeta}), t| .
$$

The latter holds for all times $t$. Let us now partition the time interval $\left[t_{i}, t_{f}\right]$ into $N+1$ equidistant pieces $\Delta t$ by writing $t_{f}-t_{i}=(N+1) \Delta t$. We can now label the intermediate times as, say $t_{n}=t_{i}+n \Delta t, n=1,2, \ldots, N$. Introducing the resolution of unity for every intermediate time point, we obtain

$$
\begin{aligned}
\left\langle 0\left(\boldsymbol{\zeta}_{f}\right), t_{f} \mid 0\left(\boldsymbol{\zeta}_{i}\right), t_{i}\right\rangle= & \left(\int_{G / H} \prod_{k=1}^{N} c d \boldsymbol{\zeta}_{k}\right)\left\langle 0\left(\boldsymbol{\zeta}_{f}\right), t_{f} \mid 0\left(\boldsymbol{\zeta}_{N}\right), t^{\prime}-\Delta t\right\rangle \\
& \times\left\langle 0\left(\boldsymbol{\zeta}_{N}\right), t^{\prime}-\Delta t \mid 0\left(\boldsymbol{\zeta}_{N-1}\right), t^{\prime}-2 \Delta t\right\rangle \\
& \times\left\langle 0\left(\boldsymbol{\zeta}_{N-1}\right), t^{\prime}-2 \Delta t \mid 0\left(\boldsymbol{\zeta}_{N-2}\right), t^{\prime}-3 \Delta t\right\rangle \\
\vdots & \times\left\langle 0\left(\boldsymbol{\zeta}_{1}\right), t+\Delta t \mid 0\left(\boldsymbol{\zeta}_{i}\right), t_{i}\right\rangle .
\end{aligned}
$$

We have formally set $t_{0}=t_{i}$ and $t_{N+1}=t_{f}$. The affiliated infinitesimal-time transition amplitude can be written as

$$
\begin{aligned}
\left\langle 0\left(\boldsymbol{\zeta}_{k}\right), t_{k} \mid 0\left(\boldsymbol{\zeta}_{k-1}\right), t_{k-1}\right\rangle \simeq\left\langle 0\left(\boldsymbol{\zeta}_{k}\right)\left|\left(1-i \int_{t_{k-1}}^{t_{k}} d t \hat{H}(t)\right)\right| 0\left(\boldsymbol{\zeta}_{k-1}\right)\right\rangle \\
\simeq\left\langle 0\left(\boldsymbol{\zeta}_{k}\right) \mid 0\left(\boldsymbol{\zeta}_{k-1}\right)\right\rangle\left(1-i \Delta t H\left(\boldsymbol{\zeta}_{k}, \boldsymbol{\zeta}_{k-1}, t_{k}\right)\right) \\
\simeq\left\langle 0\left(\boldsymbol{\zeta}_{k}\right) \mid 0\left(\boldsymbol{\zeta}_{k-1}\right)\right\rangle \exp \left(-i \int_{t_{k-1}}^{t_{k}} d t H(\boldsymbol{\zeta}, \dot{\boldsymbol{\zeta}}, t)\right)
\end{aligned}
$$

Here

$$
H\left(\boldsymbol{\zeta}_{k}, \boldsymbol{\zeta}_{k-1}, t_{k}\right)=\frac{\left\langle 0\left(\boldsymbol{\zeta}_{k}\right)\left|\hat{H}\left(t_{k}\right)\right| 0\left(\boldsymbol{\zeta}_{k-1}\right)\right\rangle}{\left\langle 0\left(\boldsymbol{\zeta}_{k}\right) \mid 0\left(\boldsymbol{\zeta}_{k-1}\right)\right\rangle},
$$

is the normalized matrix element of the Hamiltonian. Eq. (20) can be further simplified if we use the fact that to the leading order in $\Delta t$

$$
\begin{aligned}
\left\langle 0\left(\boldsymbol{\zeta}_{k}\right) \mid 0\left(\boldsymbol{\zeta}_{k-1}\right)\right\rangle & \simeq 1-\left\langle0 ( \boldsymbol { \zeta } _ { k } ) \left|\left\{\left|0\left(\boldsymbol{\zeta}_{k}\right)\right\rangle-\left|0\left(\boldsymbol{\zeta}_{k-1}\right)\right\rangle\right\}\right.\right. \\
& \simeq \exp \left(-\Delta t \frac{\left\langle0 ( \boldsymbol { \zeta } _ { k } ) \left|\left\{\left|0\left(\boldsymbol{\zeta}_{k}\right)\right\rangle-\left|0\left(\boldsymbol{\zeta}_{k-1}\right)\right\rangle\right\}\right.\right.}{\Delta t}\right) \\
& \simeq \exp \left(-\int_{t_{k-1}}^{t_{k}}\left\langle 0(\boldsymbol{\zeta})\left|\frac{d}{d t}\right| 0(\boldsymbol{\zeta})\right\rangle d t\right) .
\end{aligned}
$$


It should be also noted that both $\left|0\left(\boldsymbol{\zeta}_{j}\right)\right\rangle$ and $\left\langle 0\left(\boldsymbol{\zeta}_{i}\right)\right|$ are now the Schrödinger-picture CS. Combining Eq. (20) together with Eq. (21) allows to write the finite-time transition amplitude in the large $N$ limit as

$$
\begin{aligned}
& \left\langle 0\left(\boldsymbol{\zeta}_{f}\right), t_{f} \mid 0\left(\boldsymbol{\zeta}_{i}\right), t_{i}\right\rangle \\
& =\int_{\boldsymbol{\zeta}\left(t_{i}\right)=\boldsymbol{\zeta}_{i}}^{\boldsymbol{\zeta}\left(t_{f}\right)=\boldsymbol{\zeta}_{f}} \mathcal{D} \mu(\boldsymbol{\zeta}) \exp \left(i \int_{t_{i}}^{t_{f}} d t\left[\left\langle 0(\boldsymbol{\zeta})\left|i \frac{d}{d t}\right| 0(\boldsymbol{\zeta})\right\rangle-H(\boldsymbol{\zeta}, \dot{\boldsymbol{\zeta}}, t)\right]\right) .
\end{aligned}
$$

Here we have formally identified the functional-integral measure as

$$
\int_{\boldsymbol{\zeta}\left(t_{i}\right)=\boldsymbol{\zeta}_{i}}^{\boldsymbol{\zeta}\left(t_{f}\right)=\boldsymbol{\zeta}_{f}} \mathcal{D} \mu(\boldsymbol{\zeta}) \cdots=\lim _{N \rightarrow \infty}\left(\int_{G / H} \prod_{k=1}^{N} c d \boldsymbol{\zeta}_{k}\right) \cdots
$$

Let us also observe that the assumed square integrability of generalized CS implies

$$
\left\langle 0(\boldsymbol{\zeta})\left|i \frac{d}{d t}\right| 0(\boldsymbol{\zeta})\right\rangle=-\frac{d}{d t}\{\langle 0(\boldsymbol{\zeta})|\} i|0(\boldsymbol{\zeta})\rangle=\left(\left\langle 0(\boldsymbol{\zeta})\left|i \frac{d}{d t}\right| 0(\boldsymbol{\zeta})\right\rangle\right)^{*},
$$

i.e., $\langle 0(\boldsymbol{\zeta})|i d / d t| 0(\boldsymbol{\zeta})\rangle$ is purely real. There is an intimate connection of (24) with the concept of geometric phase. To see this we write the corresponding phase factor appearing in the path integral (22) as

$$
\int_{t_{i}}^{t_{f}}\left\langle 0(\boldsymbol{\zeta})\left|i \frac{d}{d t}\right| 0(\boldsymbol{\zeta})\right\rangle d t=\int_{\gamma}\left\langle 0(\boldsymbol{\zeta})\left|i \nabla_{\boldsymbol{\zeta}}\right| 0(\boldsymbol{\zeta})\right\rangle \cdot d \boldsymbol{\zeta}
$$

In particular, when $|0(\boldsymbol{\zeta})\rangle$ are eigenstates of the Hamiltonian (as, for instance, in nonlinear $\sigma$ models where $|0(\boldsymbol{\zeta})\rangle$ describe the degenerate ground-state) and when $\boldsymbol{\zeta}(t)$ traverses during the period $t_{f}-t_{i}$ a closed path $\gamma$ in the $G / H$ space, then (25) corresponds to the fundamental formula for the Berry-Anandan phase [10, 11, 12]. Closed paths typically occur when (quantum-mechanical) partition functions $Z$ are to be computed [1]. This is because in such a case

$$
\int_{\boldsymbol{\zeta}\left(t_{i}\right)=\boldsymbol{\zeta}_{i}}^{\boldsymbol{\zeta}\left(t_{f}\right)=\boldsymbol{\zeta}_{f}} \mathcal{D} \mu(\boldsymbol{\zeta}) \cdots \mapsto \int_{G / H} d \boldsymbol{\zeta}_{i} \int_{\boldsymbol{\zeta}\left(t_{i}\right)=\boldsymbol{\zeta}_{i}}^{\boldsymbol{\zeta}\left(t_{f}\right)=\boldsymbol{\zeta}_{i}} \mathcal{D} \mu(\boldsymbol{\zeta}) \cdots
$$

We shall say more on this in Section 5 ,

\section{2. $S U(2)$ coherent states}

Results of the previous two subsections can be now particularized for the $S U(2)$ CS. Namely, from Eq. (22) the transition amplitude can be written in the form

$$
\begin{array}{r}
\left\langle 0\left(\xi_{f}^{*}\right), t_{f} \mid 0\left(\xi_{i}\right), t_{i}\right\rangle=\lim _{N \rightarrow \infty}\left(\int \prod_{k=1}^{N} d \mu\left(\xi_{k}^{*}, \xi_{k}\right)\right) \\
\times \exp \left(i \sum_{l=0}^{N} \Delta t\left[\frac{i}{\Delta t}\left\langle 0\left(\xi_{l}^{*}\right)|\Delta| 0\left(\xi_{l}\right)\right\rangle-H\left(\xi_{l}^{*}, \xi_{l-1}, t_{l}\right)\right]\right) \\
=\int_{\xi\left(t_{i}\right)=\xi_{i}}^{\xi^{*}\left(t_{f}\right)=\xi_{f}^{*}} \mathcal{D} \mu\left(\xi^{*}, \xi\right) \exp \left(i \int_{t_{i}}^{t_{f}} d t\left[\left\langle 0\left(\xi^{*}\right)\left|i \frac{d}{d t}\right| 0(\xi)\right\rangle-H\left(\xi^{*}, \xi, t\right)\right]\right)
\end{array}
$$




$$
=\int_{\xi\left(t_{i}\right)=\xi_{i}}^{\xi^{*}\left(t_{f}\right)=\xi_{f}^{*}} \mathcal{D} \mu\left(\xi^{*}, \xi\right) \exp \left(i \int_{t_{i}}^{t_{f}} d t\left[i \frac{j\left(\xi^{*} \dot{\xi}-\dot{\xi}^{*} \xi\right)}{\left(1+|\xi|^{2}\right)}-H\left(\xi^{*}, \xi, t\right)\right]\right) .
$$

Here

$$
d \mu\left(\xi_{k}^{*}, \xi_{k}\right) \equiv \frac{d \xi_{k} d \xi_{k}^{*}}{\left(1+\left|\xi_{k}\right|^{2}\right)^{2}} \quad \text { and } \quad H\left(\xi_{l}^{*}, \xi_{l-1}, t_{l}\right) \equiv \frac{\left\langle 0\left(\xi_{l}^{*}\right)\left|H\left(t_{l}\right)\right| 0\left(\xi_{l-1}\right)\right\rangle}{\left\langle 0\left(\xi_{l}^{*}\right) \mid 0\left(\xi_{l-1}\right)\right\rangle}
$$

Use was also made of the fact that up to the order $\Delta \xi_{l}=\xi_{l}-\xi_{l-1}$ one has

$$
\left\langle 0\left(\xi_{l}^{*}\right)|\Delta| 0\left(\xi_{l}\right)\right\rangle=\left\langle0 ( \xi _ { l } ^ { * } ) \left|\left\{\left|0\left(\xi_{l}\right)\right\rangle-\left|0\left(\xi_{l-1}\right)\right\rangle\right\}=\frac{j\left(\xi_{l}^{*} \Delta \xi_{l}-\xi_{l} \Delta \xi_{l}^{*}\right)}{1+\left|\xi_{l}\right|^{2}} .\right.\right.
$$

Path integral for $S U(2)$ CS was originally constructed by Klauder [13] and Kuratsuji and Suzuki 14]. Its main utility has been in semiclassical treatments of quantum systems which have Hamiltonians composed of the generators of the $S U(2)$ group, although other applications, such as duality or geometrical phases of spin systems, are also frequently mentioned in the literature.

Generalization to field theory (e.g., to continuous spin lattice) can now proceed along standard lines. In particular one formally exchanges the coset-space variables $\zeta^{a}(t)$ $(a=1, \ldots, \operatorname{dim} G / H)$ with coset-space fields $\phi^{a}(\mathbf{x}, t)$. These fields provide a mapping from $D+1$-dimensional spacetime to group quotient $G / H$, i.e., $\phi^{a}(\mathbf{x}, t): \mathbb{R}^{D+1} \mapsto G / H$. The space $G / H$, into which the mapping is done, is known as the target space.

\section{Nambu-Goldstone theorem and the structure of vacuum manifold}

We begin this section by summarizing the quantum field theory procedure leading to the Nambu-Goldstone theorem [16, 17]. This is of course well known but it is useful to repeat it here in order to make our discussion self-contained. We will also need it in Section 5 in order to set up functional integrals for NG fields and to correctly interpret the ensuing results. Briefly stated, the theorem asserts that for a physical system with a global internal symmetry group $G$ which is spontaneously broken down to a subgroup $H$, there are $\operatorname{dim}(G / H)=\operatorname{dim} G-\operatorname{dim} H$ massless modes - NG bosons. For our purpose the best way to introduce the NG theorem is to use the Lorentz-invariant setting and apply the coset space construction of SSB [16]. A non-relativistic variant of the theorem will be discussed subsequently.

Let us assume that a full symmetry group of the system, the so-called disorderedphase symmetry, is $G$. The Hamiltonian is thus invariant under action of $G$, i.e.

$$
\hat{D}^{-1}(g) \hat{H} \hat{D}(g)=\hat{H} \quad \text { for } \quad \forall g \in G .
$$

Here $\hat{D}(g)$ is a unitary operator representing the element $g \in G$ in Hilbert space. The SSB occurs when the vacuum is invariant only under some subgroup $H$ of $G$. This, for instance, happens when the system is cooled down below a critical temperature $T_{c}$. A hallmark of the SSB is the existence of some operator $\hat{\Phi}$ known as the order parameter [18] whose ground-state expectation value $\Phi^{0}$ is not invariant under the whole group $G$, but only under $H$. The symmetry $H$ is known as the broken-phase or orderedphase symmetry. 
Let us for definiteness consider the order parameter to be a multiplet $\hat{\boldsymbol{\Phi}}$ transforming under some $n$-dimensional representation $S$ of $G$, i.e.

$$
\hat{D}^{-1}(g) \hat{\Phi}_{i} \hat{D}(g)=\sum_{j=1}^{n} S_{i j}(g) \hat{\Phi}_{j} .
$$

By definition, the vacuum expectation value $\left\langle 0\left|\hat{\Phi}_{i}\right| 0\right\rangle \equiv \Phi_{i}^{0}$ is not invariant under whole $G$ but only under $H$. This means that for $g$ from $G / H$

$$
\left\langle 0\left|\hat{D}^{-1}(g) \hat{\Phi}_{i} \hat{D}(g)\right| 0\right\rangle=\sum_{j=1}^{n} S_{i j}(g) \Phi_{j}^{0} \neq \Phi_{i}^{0} .
$$

On the level of group generators this may be phrased as

$$
\sum_{j=1}^{n} S_{i j}\left(T^{a}\right) \Phi_{j}^{0} \neq 0 \text { and } \sum_{j=1}^{n} S_{i j}\left(t^{r}\right) \Phi_{j}^{0}=0
$$

where $t^{r}$ are generators from $H$ and $T^{a}$ are broken-symmetry generators. Eq. (30) clearly shows that the ground state is not invariant under the action of $g \in G / H$, i.e.,

$$
\hat{D}(g)|0\rangle \equiv|0(g)\rangle \neq|0\rangle \text { for } g \in G / H
$$

or equivalently $\hat{D}\left(T^{a}\right)|0\rangle \neq 0$. Since states $|0(g)\rangle$ are also eigenstates of $\hat{H}$ with the same eigenvalue as $|0\rangle$ (cf. Eq. (28) ), the ground state is degenerate and distinct states are distinguished by different $g$ 's from $G / H$. So the manifold of degenerate vacuum states - vacuum manifold, can be identified with the quotient space $G / H$.

To proceed we note that (30) can be around a unit element written for all " $a$ " as

$$
\lim _{V \rightarrow \infty}\left\langle 0\left|\left[\hat{Q}_{V}^{a}(t), \hat{\Phi}_{i}(0)\right]\right| 0\right\rangle=\sum_{j=1}^{n} S_{i j}\left(T^{a}\right) \Phi_{j}^{0} \neq 0 .
$$

Here $\hat{Q}_{V}^{a}(t)$ is the regularized Noether charge associated with the generator $T^{a}$, namely

$$
\hat{Q}_{V}^{a}(t)=\int_{V} d \mathbf{x} \hat{J}_{0}^{a}(\mathbf{x}, t)
$$

where $\hat{J}_{0}^{a}(\mathbf{x}, t)$ is the conserved Noether current. In (33) we have used the translational invariance of the vacuum which allowed us to work with $\hat{\Phi}_{i}(0)$. The regularization used in Eq. (33) is necessary since $\hat{Q}^{a}$ is not mathematically well defined - it is not unitarily implementable [16]. Indeed, the translation invariance of the vacuum implies that

$$
\left\langle 0\left|\hat{Q}^{a} \hat{Q}^{a}\right| 0\right\rangle=\int d \mathbf{x}\left\langle 0\left|\hat{J}_{0}^{a}(\mathbf{x}, t) \hat{Q}^{a}\right| 0\right\rangle,
$$

is divergent. Inserting now a complete set of intermediate energy states and using again the translational invariance of the vacuum we get from (33)

$$
\begin{aligned}
\lim _{V \rightarrow \infty} \sum_{n} \int_{V} d \mathbf{x} & {\left[\left\langle 0\left|\hat{J}_{0}^{a}(0)\right| n\right\rangle\left\langle n\left|\hat{\Phi}_{i}(0)\right| 0\right\rangle e^{-i x p_{n}}\right.} \\
& \left.-\left\langle 0\left|\hat{\Phi}_{i}(0)\right| n\right\rangle\left\langle n\left|\hat{J}_{0}^{a}(0)\right| 0\right\rangle e^{i x p_{n}}\right] \\
=\sum_{n}(2 \pi)^{D} \delta^{(D)}\left(\mathbf{p}_{n}\right)\left[\left\langle 0\left|\hat{J}_{0}^{a}(0)\right| n\right\rangle\left\langle n\left|\hat{\Phi}_{i}(0)\right| 0\right\rangle e^{-i E_{n} t}\right. & \\
& \left.-\left\langle 0\left|\hat{\Phi}_{i}(0)\right| n\right\rangle\left\langle n\left|\hat{J}_{0}^{a}(0)\right| 0\right\rangle e^{i E_{n} t}\right] \neq 0 .
\end{aligned}
$$


Here $p_{n}=\left(E_{n}, \mathbf{p}_{n}\right)$ and $D$ is the spatial dimension. As long as the theory satisfies the microcausality condition, i.e., the commutator of any two local operators separated by a space-like interval vanishes, we have

$$
\begin{aligned}
\frac{d}{d t}\left[\hat{Q}_{V}^{a}(t), \hat{\Phi}_{i}(0)\right] & =\int_{V} d \mathbf{x}\left[\partial^{\mu} \hat{J}_{\mu}^{a}(\mathbf{x}, t), \hat{\Phi}_{i}(0)\right] \\
& -\oint_{\Sigma} d S^{i}\left[\hat{J}_{i}^{a}(\mathbf{x}, t), \hat{\Phi}_{i}(0)\right] \stackrel{V \rightarrow \infty}{\longrightarrow} 0 .
\end{aligned}
$$

$\Sigma$ denotes the surface bounding the volume $V$, i.e. the sphere $S^{D-1}$. This indicates that after the time derivative the last two lines of (36) give

$$
\begin{aligned}
& \sum_{n}(2 \pi)^{D} \delta^{(D)}\left(\mathbf{p}_{n}\right) E_{n} {\left[\left\langle 0\left|\hat{J}_{0}^{a}(0)\right| n\right\rangle\left\langle n\left|\hat{\Phi}_{i}(0)\right| 0\right\rangle e^{-i E_{n} t}\right.} \\
&\left.+\left\langle 0\left|\hat{\Phi}_{i}(0)\right| n\right\rangle\left\langle n\left|\hat{J}_{0}^{a}(0)\right| 0\right\rangle e^{i E_{n} t}\right]=0 .
\end{aligned}
$$

Comparing (36) with (38) shows that there must exist a state $|n\rangle$ such that

$$
\left\langle 0\left|\hat{\Phi}_{i}(0)\right| n\right\rangle\left\langle n\left|\hat{J}_{0}^{a}(0)\right| 0\right\rangle \neq 0 \quad \text { for } \quad \delta^{(D)}\left(\mathbf{p}_{n}\right) E_{n}=0 .
$$

This state is a massless state with the same quantum number as $\hat{Q}^{a}$ since it is generated by $\hat{Q}^{a}$ from the vacuum $|0\rangle$. In particular, the field excitations corresponding to this state (the so-called the NG excitations) must have the same Lorentz properties as the charge $\hat{Q}^{a}$. Because the charge is related to internal symmetries, the NG field must be a Lorentz scalar (or pseudo-scalar) and a boson. A similar argument for spontaneouslybroken supersymmetry implies that the NG particles are spin- $1 / 2$ fermions, and they are spin-1 bosons (e.g., phonons) for spontaneously-broken translation invariance.

Let us define the vacuum state $|0(\boldsymbol{\pi})\rangle \equiv \exp (i \boldsymbol{\pi} \cdot \hat{Q})|0\rangle$ where $\boldsymbol{\pi} \cdot \hat{Q}=\pi_{a} \hat{Q}^{a}$. If we consider in the neighborhood of the vacuum state $|0(\pi)\rangle$ an infinitesimal transformation $\boldsymbol{\theta}$, say in the direction " $a$ ", we obtain (no summation over " $a$ ")

$$
\delta_{\theta}|0(\boldsymbol{\pi})\rangle=\exp \left(i \theta_{a} \hat{Q}^{a}\right)|0(\boldsymbol{\pi})\rangle-|0(\boldsymbol{\pi})\rangle=i \theta_{a} \hat{Q}^{a}|0(\boldsymbol{\pi})\rangle .
$$

Because the argument leading to (39) could be repeated for any ground state $|0(g)\rangle$, $g \in G / H$, Eq. (40) implies that $\delta|0(\boldsymbol{\pi})\rangle \propto|n\rangle$ for any $\boldsymbol{\pi}$. So the NG state corresponds to a shift within the vacuum manifold (shift along "flat energy directions"). In this respect, the NG fields give a meaning to the fluctuations among degenerate ground states. Note that the field which $\delta_{\theta}$-fluctuates in $a$-th energy flat direction can be associated with the group parameter $\theta_{a}$. One may thus identify the local group parameters $\boldsymbol{\theta}$ with the NG multiplet. Since at every point $\pi$ of the vacuum manifold there are $\operatorname{dim}(G / H)$ independent flat directions (namely independent tangent directions of the local frame in $\boldsymbol{\pi}$ ), there must be $\operatorname{dim}(G / H)$ distinct NG fields. So $\boldsymbol{\theta}$ form a local coordinate system at $\boldsymbol{\pi}$. Starting with a fixed $\boldsymbol{\pi}$, one may extend the local domain of $\boldsymbol{\theta}$ globally on the whole $G / H$ by applying the transformation rules for broken symmetries in $G / H$ on the parameters $\boldsymbol{\theta}$. The involved mathematical technicalities are most easily done through the Maurer-Cartan one-forms [19]. Extension of the NG fields on the whole $G / H$ allows to put in one-to-one connection the NG fields and points on $G / H$. In this way, the NG fields coordinatize the quotient space $G / H$. 
Alternatively, one may view the NG modes as representing the fluctuations in the order parameter. Indeed, using (for a simplicity of the argument) the vacuum state at $\boldsymbol{\pi}=0$ we can write (no summation over " $a$ ")

$$
\lim _{V \rightarrow \infty}\left\langle 0\left|i \theta_{a}\left[\hat{Q}_{V}^{a}(t), \hat{\Phi}_{i}(0)\right]\right| 0\right\rangle=\delta \Phi_{i}^{0}=i \theta_{a} S_{i j}\left(T^{a}\right) \Phi_{j}^{0} .
$$

From our previous discussion follows that the local parameter $\theta_{a}$ coincides with the near-to-origin NG field, and so the $\delta \Phi_{i}^{0}$ is directly proportional to the NG field. The preceding equation is often a reason why some people normalize the NG field in such a way that $\theta_{a} S_{i j}\left(T^{a}\right) \Phi_{j}^{0}$ itself is considered as the definition of the NG field [19].

As shown in Section 2, the group quotient $G / H$ can be identified with a set of all generalized CS corresponding to the group $G$. Connection with a vacuum manifold is then established when as a fiducial vector one chooses any ground-state vector $|0(g)\rangle$.

Let us finally stress that the NG theorem is valid, with few qualifications, even for non-Lorentz-invariant situations such as those that occur frequently in solid-state physics. The caveat in the above proof is the use of translational invariance and

microcausality. In particular the microcausality should be in the non-relativistic setting substituted with an absence of long-range interactions [20]. Under assumption that the translational invariance is not broken it can be showed that the total number of NG bosons might be less than the number of broken generators, in contrast to the naive expectation based on experience with Lorentz-invariant systems. The precise rule for counting the NG modes can be found, e.g., in Ref. [20].

Fortunately the NG fields serve also in the non-relativistic framework as coordinates on the vacuum manifold $G / H$. The point is that the number of NG fields still coincide with the number of broken generators, it is only that the number of NG fields does not match the number of NG bosons. Connection between broken generators and NG bosons depends in a non-relativistic context on the dispersion relation. This will be explicitly illustrated in the following section.

\section{5. $S U(2) / U(1)-\sigma$ model and Landau-Lifshitz ferromagnetic magnons}

Because the functional integrals based on generalized coherent states are naturally phrased in terms of coset-space variables they are well suited to describe the effective low-energy dynamics of theories with SSB. In particular, when $G$ is the disorderedphase symmetry and $H$ is the broken-phase symmetry then the NG fields take values in the target space which is a coset of groups $G / H$. More details can be found, e.g., in Ref. [16]. Massless field theories where the target space is the group coset space $G / H$ are commonly known as $G / H-\sigma$ models or also as non-linear $\sigma$ models. With a suitable choice of the Hamiltonian $H(\boldsymbol{\zeta}, \dot{\zeta}, t)$ will the generalized CS functional integrals (and the associated non-linear $\sigma$ models) describe low-energy effective field theories, in which only NG bosons, including their mutual interactions, will propagate.

NG bosons are true dynamical protagonists in many low-energy or low-temperature solid-state systems. In this respect it is instructive to consider some representative 
system where one can explicitly see how the correct NG dynamics is reproduced via generalized CS path integrals. Along these lines we now derive the correct behavior of ferromagnetic magnons in the Heisenberg model of ferromagnets. This problem was historically notoriously difficult to deal with. In particular, the usual mean-field approaches fail to provide the quadratic dispersion behavior which is typically observed in inelastic scattering of spin-polarized neutrons by magnons. Since ferromagnetic materials are paradigmatic examples of systems with SSB [16] — the disordered-phase symmetry $S U(2)$ is below the Curie temperature spontaneously broken to the residual rotational symmetries $U(1)$ — it is only natural to use the $S U(2) / U(1)-\sigma$ model to deal with the corresponding low-energy degrees of freedom. The resulting gapless NG modes should be then identifiable with scalar bosonic excitations around the ground state of the spin- $j$ Heisenberg ferromagnets. The only experimentally viable candidates for such excitations are the gapless spin waves known as magnons. By following this reasoning we show that in the long-wavelength limit one can obtain the Landau-Lifshitz non-linear $\sigma$ model which describes the correct dynamics and dispersion relations for ferromagnetic magnons.

To see how all this comes about we first rewrite the action in the path integral (27) in terms of the unit-vector dynamical variables $\mathbf{n}(t)$. The first term can be then expressed as

$$
\begin{aligned}
i \frac{j\left(\xi^{*} d \xi-d \xi^{*} \xi\right)}{\left(1+|\xi|^{2}\right)} & =-2 j \sin ^{2}(\theta / 2) d \varphi=-\frac{j}{r(z+r)}(x d y-y d x) \\
& =\mathbf{A}_{B}(\mathbf{x}) \cdot d \mathbf{x}
\end{aligned}
$$

where the vector potential

$$
\mathbf{A}_{B}(\mathbf{x})=-\frac{j}{r(z+r)}(-y, x, 0)
$$

corresponds to Berry's connection. Since the vector $\mathbf{x}$ sweeps the surface of $\mathcal{S}^{2}$ we have that $\mathbf{x}=\mathbf{n}\left(\mathbf{n}^{2}=1\right)$. The first term in the action in (27) thus reads

$$
i \int_{t_{i}}^{t_{f}} d t \frac{j\left(\xi^{*} \dot{\xi}-\dot{\xi}^{*} \xi\right)}{\left(1+|\xi|^{2}\right)}=\int_{t_{i}}^{t_{f}} \mathbf{A}_{B}(\mathbf{n}) \cdot \frac{d \mathbf{n}}{d t} d t=\int_{\Sigma} \mathbf{B}_{B} \cdot d \boldsymbol{\sigma} .
$$

With $\Sigma$ denoting area of $\mathcal{S}^{2}$ bounded by a closed loop traversed by $\mathbf{n}(t)$. Berry's magnetic induction $\mathbf{B}_{B}$ has the explicit form

$$
\mathbf{B}_{B}(\mathbf{x})=\nabla \wedge \mathbf{A}_{B}(\mathbf{x})=\frac{j}{r^{3}} \mathbf{x}=\frac{j}{r^{2}} \mathbf{n}=j \mathbf{n},
$$

which implies that

$$
\int_{\mathcal{S}^{2}} \mathbf{B}_{B} \cdot d \boldsymbol{\sigma}=4 \pi j
$$

Eq. (45) together with (46) shows that there is a monopole of the magnetic charge $j$ located in the origin of our target space. We also note that from (44) and (45) follows

$$
\begin{aligned}
i \int_{t_{i}}^{t_{f}} d t \frac{\left(\xi^{*} \dot{\xi}-\dot{\xi}^{*} \xi\right)}{\left(1+|\xi|^{2}\right)} & =\int_{0}^{1} d u \int_{t_{i}}^{t_{f}} d t \mathbf{n}(t, u) \cdot\left[\partial_{t} \mathbf{n}(t, u) \wedge \partial_{u} \mathbf{n}(t, u)\right] \\
& \equiv S_{W Z}[\mathbf{n}]
\end{aligned}
$$


where $\mathbf{n}(t, u)$ is an arbitrary extension of $\mathbf{n}(t)$ into the spherical rectangle defined by the limits of integration and fulfilling conditions: $\mathbf{n}(t, 0)=\mathbf{n}(t), \mathbf{n}(t, 1)=(1,0,0)$, and $\mathbf{n}\left(t_{i}, u\right)=\mathbf{n}\left(t_{f}, u\right)$. The $S_{W Z}[\mathbf{n}]$ is a special member of a wide class of actions known as Wess-Zumino actions [15]. Eq. (47) then demonstrates a typical situation ubiquitous in effective theories, namely, that Berry-Anandan phase gives rise to the Wess-Zumino action. Examples include low-dimensional ferromagnets with local anisotropies [21] or non-abelian gauge theories with topological angle $(\theta$-term) [22].

Let us now turn to many-spin systems and consider a lattice of spins. We will concentrate first on the Hamiltonian $H\left(\xi^{*}, \xi, t\right)$. To this end we consider the Hamiltonian for the ferromagnetic Heisenberg model, i.e.

$$
\hat{H}(\mathbf{J})=K \sum_{\left\{\mathbf{x}, \mathbf{x}^{\prime}\right\}} \hat{\mathbf{J}}(\mathbf{x}) \cdot \hat{\mathbf{J}}\left(\mathbf{x}^{\prime}\right) .
$$

where $K=-|K|$ is the Heisenberg exchange constant and $\left\{\mathbf{x}, \mathbf{x}^{\prime}\right\}$ denotes pairs of neighboring lattice sites. According to the definition of $H\left(\xi_{k}^{*}, \xi_{k-1}, t\right)$ we have

$$
\begin{aligned}
H\left(\xi_{k}^{*}, \xi_{k-1}, t\right) & =H\left(\mathbf{n}_{k}, \mathbf{n}_{k-1}\right)=\frac{\left\langle 0\left(\mathbf{n}_{k}\right)|\hat{H}(\mathbf{J})| 0\left(\mathbf{n}_{k-1}\right)\right\rangle}{\left\langle 0\left(\mathbf{n}_{k}\right) \mid\left(\mathbf{n}_{k-1}\right)\right\rangle} \\
& \approx\left\langle 0\left(\mathbf{n}_{k}\right)|\hat{H}(\mathbf{J})| 0\left(\mathbf{n}_{k}\right)\right\rangle+\mathcal{O}(\Delta t) .
\end{aligned}
$$

By taking advantage of the identity $\left\langle 0\left(\mathbf{n}_{k}\right)|\hat{\mathbf{J}}(\mathbf{x})| 0\left(\mathbf{n}_{k}\right)\right\rangle=j \mathbf{n}_{k}(\mathbf{x})$ we obtain

$$
H\left(\mathbf{n}_{k}, \mathbf{n}_{k-1}\right) \approx-|K| j^{2} \sum_{\left\{\mathbf{x}, \mathbf{x}^{\prime}\right\}} \mathbf{n}_{k}(\mathbf{x}) \cdot \mathbf{n}_{k}\left(\mathbf{x}^{\prime}\right)
$$

so that action in the functional integral (27) reads

$$
S[\mathbf{n}]=j \sum_{\mathbf{x}} S_{W Z}[\mathbf{n}(\mathbf{x})]+|K| j^{2} \sum_{k} \Delta t \sum_{\left\{\mathbf{x}, \mathbf{x}^{\prime}\right\}} \mathbf{n}_{k}(\mathbf{x}) \cdot \mathbf{n}_{k}\left(\mathbf{x}^{\prime}\right) .
$$

Here the first sum runs over all the sides of the lattice and thus represents the sum of the Wess-Zumino terms of individual spins. Note particularly, that the time derivative (and hence dynamics) enters only through the Wess-Zumino term.

For definiteness sake we now consider a $D$-dimensional hypercubic lattice and restrict $\sum_{\left\{\mathbf{x}, \mathbf{x}^{\prime}\right\}}$ to nearest neighbors only. With this we can write

$$
\sum_{\left\{\mathbf{x}, \mathbf{x}^{\prime}\right\}} \mathbf{n}_{k}(\mathbf{x}) \cdot \mathbf{n}_{k}\left(\mathbf{x}^{\prime}\right)=-\frac{1}{2} \sum_{\left\{\mathbf{x}, \mathbf{x}^{\prime}\right\}}\left[\mathbf{n}_{k}(\mathbf{x})-\mathbf{n}_{k}\left(\mathbf{x}^{\prime}\right)\right]^{2}+\text { const. }
$$

Consider now the long-wavelength limit, in which $\mathbf{n}_{k}(\mathbf{x})$ are smooth functions of $\mathbf{x}$. By denoting the lattice spacing $a$ and taking the $N \rightarrow \infty$ (i.e., continuous-time) limit we obtain an effective field theory described by the action

$$
\begin{aligned}
S[\mathbf{n}] & =\frac{j}{a^{D}} \int_{\mathbb{R}^{D}} d^{D} \mathbf{x} S_{W Z}[\mathbf{n}(\mathbf{x})] \\
& -\frac{j^{2}|K|}{2 a^{D-2}} \int_{t_{i}}^{t_{f}} d t \int_{\mathbb{R}^{D}} d^{D} \mathbf{x} \partial_{i} \mathbf{n}(\mathbf{x}, t) \cdot \partial_{i} \mathbf{n}(\mathbf{x}, t) .
\end{aligned}
$$

In this expression we have dropped the constant term from (52) which is irrelevant for dynamical equations. In order to deal with the non-trivial measure $\mathcal{D} \mu(\mathbf{n})$ in the 
functional integral, we can rewrite it as $\mathcal{D} \mu(\mathbf{n}) \delta\left[\mathbf{n}^{2}-1\right]$ where the integration variables $\mathbf{n}$ are not any more restricted to a target space $\mathcal{S}^{2}$. The functional $\delta$-function can be elevated into the action via functional Fourier transform

$$
\begin{aligned}
\delta\left[\mathbf{n}^{2}-1\right] & =\lim _{N \rightarrow \infty} \prod_{i=1}^{N} \delta\left(\mathbf{n}^{2}\left(\mathbf{x}_{i}, t_{i}\right)-1\right) \\
& =\int \mathcal{D} \lambda \exp \left(i \int_{t_{i}}^{t_{f}} d t \int_{\mathbb{R}^{D}} d^{D} \mathbf{x} \lambda(\mathbf{x}, t)\left(\mathbf{n}^{2}(\mathbf{x}, t)-1\right)\right) .
\end{aligned}
$$

The latter leads to a new total action

$$
S_{\mathrm{tot}}[\mathbf{n}]=S[\mathbf{n}]+\int_{t_{i}}^{t_{f}} d t \int_{\mathbb{R}^{D}} d^{D} \mathbf{x} \lambda(\mathbf{x}, t)\left(\mathbf{n}^{2}(\mathbf{x}, t)-1\right) .
$$

Let us now look at the classical equation of motion whose solution should represent the dominant field configuration in a semiclassical WKB approach to quantum ferromagnetism. The variation $\delta S_{\text {tot }}[\mathbf{n}]=0$ implies three equations

$$
j\left(\mathbf{n} \wedge \partial_{t} \mathbf{n}\right)+2 a^{D} \lambda \mathbf{n}=-a^{2}|K| j^{2} \nabla^{2} \mathbf{n} \quad \text { and } \quad \mathbf{n}^{2}=1 .
$$

Here we have employed that

$$
\begin{aligned}
\delta S_{W Z}[\mathbf{n}(\mathbf{x})] & =\int_{0}^{1} d u \int_{t_{i}}^{t_{f}} d t \partial_{u}\left\{\delta \mathbf{n}(\mathbf{x}, t, u) \cdot\left[\mathbf{n}(\mathbf{x}, t, u) \wedge \partial_{t} \mathbf{n}(\mathbf{x}, t, u)\right]\right\} \\
& +3 \int_{0}^{1} d u \int_{t_{i}}^{t_{f}} d t \delta \mathbf{n}(\mathbf{x}, t, u) \cdot\left[\partial_{t} \mathbf{n}(\mathbf{x}, t, \tau) \wedge \partial_{u} \mathbf{n}(\mathbf{x}, t, u)\right] \\
& =\int_{t_{i}}^{t_{f}} d t \delta \mathbf{n}(\mathbf{x}, t) \cdot\left[\mathbf{n}(\mathbf{x}, t) \wedge \partial_{t} \mathbf{n}(\mathbf{x}, t)\right],
\end{aligned}
$$

where the term on the second line is zero because $\partial_{t} \mathbf{n} \wedge \partial_{u} \mathbf{n}$ is parallel to $\mathbf{n}$ and $\mathbf{n} \cdot \delta \mathbf{n}=\delta \mathbf{n}^{2} / 2=0$. On the last line we have used that $\mathbf{n}(t, 0)=\mathbf{n}(t), \mathbf{n}(t, 1)=(1,0,0)$. Employing now the identity $\mathbf{n} \cdot\left(\mathbf{n} \wedge \partial_{t} \mathbf{n}\right)=0$ we find for the Lagrange multiplier $\lambda$

$$
\lambda=-\frac{|K| j^{2}}{2 a^{D-2}} \mathbf{n} \cdot \nabla^{2} \mathbf{n} .
$$

By inserting this result back into Eq. (56) and applying the identity $\mathbf{a} \wedge(\mathbf{b} \wedge \mathbf{c})=$ $(\mathbf{a} \cdot \mathbf{c}) \mathbf{b}-(\mathbf{a} \cdot \mathbf{b}) \mathbf{c}$ we obtain

$$
\mathbf{n} \wedge\left[\partial_{t} \mathbf{n}-a^{2}|K| j\left(\mathbf{n} \wedge \nabla^{2} \mathbf{n}\right)\right]=0 .
$$

Note that both terms inside [...] are orthogonal to $\mathbf{n}$ and so we can cast the previous equation into a simpler (but equivalent) form, namely

$$
\partial_{t} \mathbf{n}=a^{2}|K| j\left(\mathbf{n} \wedge \nabla^{2} \mathbf{n}\right) .
$$

Eq. (60) is known as Landau-Lifshitz equation for quantum ferromagnet [18]. It essentially describes the dynamics of a ferromagnetic spin wave. To see a leading dispersion behavior, we go to the linear regime and assume that the spins are align around a 3-rd axis around which they wobble, or precess, so in particular $n_{3}$ will change with $t$ and $\mathbf{x}$ much slower that $n_{1,2}$. By defining, $\mathbf{n}=\left(\pi_{1}, \pi_{2}, \sigma\right)\left(\boldsymbol{\pi}^{2}+\sigma^{2}=1\right)$, omitting derivatives of $\sigma$ and setting $\sigma \approx 1$ we linearize the Landau-Lifshitz equations as

$$
\partial_{t} \pi_{1} \approx-a^{2}|K| j \nabla^{2} \pi_{2} \text { and } \partial_{t} \pi_{2} \approx a^{2}|K| j \nabla^{2} \pi_{1}
$$


Fourier transform of (61) yields the dispersion relation $\omega(\mathbf{k}) \propto \mathbf{k}^{2}$. The modes that obey such a behavior are ferromagnetic magnons. These are true (non-relativistic) NambuGoldstone bosons. However, notice that the fields $\pi_{1}$ and $\pi_{2}$ describe only one NG mode. This can be understood by rewriting (61) equivalently as

$$
\partial_{t} \pi \approx i a^{2}|K| j \nabla^{2} \pi \text { and } \partial_{t} \pi^{\dagger} \approx-i a^{2}|K| j \nabla^{2} \pi^{\dagger},
$$

with $\pi=\pi_{1}+i \pi_{2}$. Since the fields satisfy first order equations, $\pi$ must contain only annihilation operators and $\pi^{\dagger}$ only creation operators. So we need two NG fields for describing a physical particle (the NG boson). With (61) and 62) we have recovered the well known experimental result (see, e.g. Ref. [23]) that the dispersion relation of ferromagnetic spin waves has a non-relativistic form. Note that Berry-Anandan phase was essential in obtaining the right dispersion relation.

The functional integral (27) with the action (55) represents a particular class of nonlinar $\sigma$ models known as Landau-Lifshitz $\sigma$ models. In general, the Landau-Lifshitz $\sigma$ models are models defined on a general coset space $G / H$, with $H$ a maximal stability sub-group of $G$. These are non-relativistic models that have $G$-valued Noether charges, local $H$ invariance and are classically integrable.

Similar analysis can be performed also for anti-ferromagnets, e.g., along the lines proposed in Ref. [24]. In this case the classical lowest energy configuration is described by the Néel state [23] where the neighboring lattice spins flip the sign, i.e. $\mathbf{n}(l) \mapsto(-1)^{l} \mathbf{n}(l)$. The result of absorbing this sign flip is that $H(\mathbf{x}, \dot{\mathbf{x}}, t)$ and every other $S_{W Z}[\mathbf{n}(\mathbf{x})]$ (i.e., Wess-Zumino term of the individual spins) change sign. With this one can show that the dispersion relation of spin waves have the linear (relativistic-like) form $\omega(\mathbf{k}) \propto|\mathbf{k}|$. This linear gapless dispersion describes the relativistic-like Nambu-Goldstone modes, which are in this case called anti-ferromagnetic magnons. It is interesting to point out that in anti-ferromagnets the corresponding Berry-Anandan phase does not play a dynamical role because in the Néel state the Wess-Zumino term reduces to a topological charge [24].

\section{Final notes}

Let us end up with a few notes concerning the presented approach. We have shown that the functional integrals for $G / H-\sigma$ models which account for quantum dynamics of NG bosons (i.e., gapless excitations that live in the broken phase of spontaneously broken systems) can be naturally phrased in terms of generalized CS functional integrals. As we have seen, this is because the NG fields take their values in the target space which is the group quotient space $G / H$. Group $G$ in the question is the symmetry of the original (disordered) phase, while $H$ is the residual symmetry after spontaneous symmetry breakdown. State vectors that characterize such NG excitations are then inevitably labeled by points from $G / H$. With a suitable choice of a fiducial state they can be identified with a group- $G$ related CS.

An interesting byproduct of the CS functional integrals is that they naturally generate a Berry-Anandan phase. From Eq. (21) we have seen that the Berry-Anandan 
phase is determined by the overlaps, i.e., by the inner products, between CS. Essential in this case is that representations of CS are square-integrable. Mathematically the Berry-Anandan phase represents anholonomy with respect to the natural (Berry's) connection along a closed loop in the projective Hilbert space [11. For CS such a non-trivial anholonomy reflects the "frustration" of assigning a common phase to all of CS along a closed path in a parameter space [16, 24]. Closed paths in a parameter space appear typically in the formulation of the partition function. In cases when transition amplitudes are considered one should work with Pancharatnam's phase instead [12]. Since the Berry-Anandan phase enters into the action of the CS functional integral it might affect the dynamical properties of the system. In particular, it can (and often it does) change dynamical equations and dispersion relations of the associated NG excitations.

We have illustrated the aforementioned connection between non-linear $\sigma$ models and group-related CS with a spin- $j$ Heisenberg ferromagent in a broken phase. Apart from the correct dynamical Landau-Lifshitz equations for quantum ferromagnet we have obtained also correct linear dispersion relation for ferromagnetic magnons. This was possible only because the Berry-Anandan phase exemplified via Wess-Zumino term furnished the dynamical equations with the first time-derivative term. It should be further noted that the exact form of the dispersion relation could not be specified by Goldstone's theorem alone. Dispersion relations are not determined merely by symmetry considerations, they also crucially depend on the specifics of the system, namely on the choice of the Hamiltonian $H(\mathbf{x}, \dot{\mathbf{x}}, t)$ which specifies the actual interaction between NG fields and on the spin orientations in respective sublattices which determines type of spin waves (ferromagnetic or anti-ferromagnetics) and hence type of the NG field. It is also important to observe that even if we have the same symmetry breaking pattern $S U(2) \rightarrow$ $U(1)$, the ferromagnetic and anti-ferromagnetic systems differ in their qualitative description of the dispersion relation. For instance, the number of independent magnon states differs [23]; one for a ferromagnet and two for anti-ferromagnet. In fact, only the number of real NG fields turns out to be universal and equals to the dimension of the coset space $S U(2) / U(1)$ which is $\operatorname{dim}[S U(2)]-\operatorname{dim}[U(1)]=2$ (for ferromagnets these are fields $\pi_{1}$ and $\pi_{2}$ ).

Let us also note that in the large $j$ limit is the $S U(2)$ CS functional integral dominated by the stationary points of $S_{\text {tot }}[\mathbf{n}]$, i.e. by solutions of Eq. (59). In fact, with increasing $j$ will the semiclassical representation of the above $S U(2)$ CS functional integral approximate the exact partition function. For this reason one might arrange the semiclassical result as power series in $1 / j$ in much the same way as the $1 / N$ perturbation expansion is done, e.g., in $O(N)$ symmetric models. Such an expansion is known as the Holstein-Primakoff expansion [25]. 


\section{Acknowledgments}

A particular thanks go to G. Vitiello, H. Kleinert and J. Tolar for enlightening discussions, and to T.W.B. Kibble, J. Klauder and G. Junker for their constructive feedbacks. M.B. is supported by MIUR and INFN. P.J. is supported by the Ministry of Education of the Czech Republic under the Grant No. CFRJS 1507001.

\section{References}

[1] Kleinert H 2009 Path Integrals in Quantum Mechanics, Statistics, Polymer Physics, and Financial Markets (London: World Scientific)

[2] Zinn-Justin J 2010 Path Integrals in Quantum Mechanics (Oxford: Oxford University Press)

[3] Perelomov A M 1986 Generalized coherent states and their applications (Berlin: Springer Verlag)

[4] Arecchi F T, Courtens E, Gilmore R and Thomas H 1972 Phys. Rev. A 62211

[5] D'Ariano G and Rasetti M 1985 Phys. Lett. A 107291

[6] D'Ariano G, Rasetti M and Vadacchino M 1985 J. Phys. A 181295

[7] Rasetti M 1975 Int. J. Theor. Phys. 141

[8] Ali S T, Antoine J-P and Gazeau J-P 2000 Coherent States, Wavelets and Their Generalizations (Berlin: Springer Verlag)

[9] Gazeau J-P 2009 Coherent States in Quantum Physics (Berlin: Wiley-VCH)

[10] Berry M V 1984 Proc. Roy. Soc. Lond. A 39245

[11] Aharonov Y and Anandan J 1987 Phys. Rev. Lett. 581593

[12] Blasone M and Jizba P 2004 Ann. Phys., NY 312354

[13] Klauder J R 1979 Phys. Rev. D 192349

[14] Kuratsuji H and Suzuki T 1980 J. Math. Phys. 21472

[15] Witten E 1983 Nucl. Phys. B 223422

[16] Blasone M, Jizba P and Vitiello G 2011 Quantum Field Theory and its Macroscopic Manifestations (London: Imperial College Press)

[17] Goldstone J, Salam A and Weinberg S 1962 Phys. Rev. 127648

[18] Landau L D and Lifshitz E M 1991 Statistical Mechanics, Part 1 (Oxford: Pergamon Press)

[19] Burgess C P 2000 Phys. Rep. 330193

[20] Nielsen H B and Chadha S 1076 Nucl. Phys. B 105445

[21] Braun H-B and Loss D 1996 Phys. Rev. B 533237

[22] Altland A and Simons B D 2010 Condensed Matter Field Theory (Cambridge: CUP)

[23] Ashcroft N W and Mermin N D 1976 Solid State Physics (Philadelphia: Harcourt College)

[24] Fradkin E and Stone M 1988 Phys. Rev. B 38721

[25] Holstein T and Primakoff H 1940 Phys. Rev. 581098 